International Journal of Pure and Applied Mathematics

Volume 103 No. 1 2015, 19-26

ISSN: 1311-8080 (printed version); ISSN: 1314-3395 (on-line version)

url: http://www.ijpam.eu

doi: http://dx.doi.org/10.12732/ijpam.v103i1.2

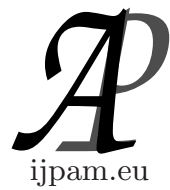

\title{
ON $b$-CONVERGENCE OF $p$-STACKS
}

\author{
N. Karthikeyan ${ }^{1} \S$, N. Rajesh ${ }^{2}$ \\ ${ }^{1}$ Department of Mathematics \\ Jeppiaar Engineering College \\ Chennai, 600119, Tamilnadu, INDIA \\ ${ }^{2}$ Department of Mathematics \\ Rajah Serfoji Govt. College \\ Thanjavur, 613005, Tamilnadu, INDIA
}

\begin{abstract}
We introduce the notion of $b$-convergence of $p$-stacks and by using that notion we characterize the $b$-interior, $b$-closure, separation axioms and $b$-irresoluteness on a topological space. Also we introduce a new notion of $p$ - $b$ compactness and investigate its properties in terms of $b$-convergence of $p$-stacks.
\end{abstract}

AMS Subject Classification: 54C10, 54C08, 54C05

Key Words: topological spaces, $b$-open sets, $b$-closed spaces

\section{Introduction}

Generalized open sets play a very important role in General Topology and they are now the research topics of many topologists worldwide. Indeed a significant theme in General Topology and Real analysis concerns the various modified forms of continuity, separation axioms etc. by utilizing generalized open sets. In 1996, Andrijevic [1] introduced a new class of generalized open sets called bopen sets into the field of topology. Andrijevic studied several fundamental and interesting properties of $b$-open sets. In this paper, we introduce the notion of $b$ -

Received: January 23, 2015

(C) 2015 Academic Publications, Ltd.

$\S_{\text {Correspondence author }}$ url: www.acadpubl.eu 
convergence of $p$-stacks and by using that notion we characterize the $b$-interior, $b$-closure, separation axioms and $b$-irresoluteness on a topological space. Also we introduce a new notion of p-b-compactness and investigate its properties in terms of $b$-convergence of $p$-stacks.

\section{Preliminaries}

Throughout this paper, spaces always means topological spaces on which no separation axioms are assumed unless otherwise mentioned and $f:(X, \tau) \rightarrow$ $(Y, \sigma)$ (or simply $f: X \rightarrow Y$ ) denotes a function $f$ of a space $(X, \tau)$ into a space $(Y, \sigma)$. Let $A$ be a subset of a space $X$. The closure and the interior of $A$ are denoted by $\operatorname{Cl}(A)$ and $\operatorname{Int}(A)$, respectively. A subset $A$ of $X$ is said to be $b$-open [1] (= $\gamma$-open [4]) $A \subset \operatorname{Int}(\operatorname{Cl}(A)) \cup \mathrm{Cl}(\operatorname{Int}(A))$. The complement of a $b$-open set is called a $b$-closed set [1] $(=\gamma$-closed set [4])). The union of all $b$-open sets contained in $A \subset X$ is called the $b$-interior of $A$, and is denoted by $b \operatorname{Int}(A)$. The intersection of all $b$-closed sets containing $A$ is called the $b$-closure [1] of $A$ and is denoted by $b \mathrm{Cl}(A)$. A subset $M(x)$ of a topological space $X$ is called a $b$-neighbourhood of a point $x \in X$ if there exists a $b$-open set $S$ such that $x \in S \subset M(x)$. Given a set $X$, a collection $\mathbf{C}$ of subsets of $X$ is called a stack if $A \in \mathbf{C}$ whenever $B \in \mathbf{C}$ and $B \subset A$. A stack $\mathbf{H}$ on a set $X$ is called a $p$-stack if it satisfies the following condition: $(P) A, B \in \mathbf{H} \Rightarrow A \cap B \neq \emptyset$. Condition $(P)$ is called the pairwise intersection property (P.I.P). A collection B of subsets of $X$ with the P.I.P is called a $p$-stack base. For any collection $B$, we denote by $<\mathbf{B}>=\{A \subset X$ : there exists $B \in \mathbf{B}$ such that $B \subset A\}$ the stack generated by $\mathbf{B}$, and if $\{B\}$ is a $p$-stack base, then $\langle\{B\}>$ is a $p$-stack. We will denote simply $\langle\{B\}\rangle=\langle B\rangle$. In case $x \in X$ and $B=\{x\},\langle x\rangle$ is usually denoted by $x$. Let $p S(X)$ denote the collection of all $p$-stacks on $X$, partially ordered by inclusion. The maximal elements in $p S(X)$ are called ultra $p$-stacks is contained in an ultra $p$-stack. For a function $f: X \rightarrow Y$ and $\mathbf{H} \in p S(X)$, the image stack $f(\mathbf{H})$ in $p S(Y)$ has $p$-stack base $\{f(H): H \in \mathbf{H}\}$. Likewise, if $\mathbf{G} \in p S(Y), f^{-1}(\mathbf{G})$ denotes the $p$-stack on $X$ generated by $\left\{f^{-1}(G): G \in \mathbf{G}\right\}$.

Definition 2.1. Let $(X, \tau)$ be a topological space. A class $G_{i}$ of $b$-open subsets of $X$ is said to be $b$-open cover of $X$ if each point in $X$ belongs to at least one $G_{i}$ that is $\underset{i}{\cup} G_{i}=X$.

Definition 2.2. A subset $K$ of a nonempty set $X$ is said to be $b$-compact relative to $(X, \tau)$ if every cover of $K$ by $b$-open sets of $X$ has a finite subcover. 
We say that $(X, \tau)$ is $b$-compact if $X$ is $b$-compact.

Definition 2.3. A topological space $(X, \tau)$ is said to be:

(i) $b$ - $T_{1}$ [2] if for each pair of distinct points $x$ and $y$ of $X$, there exist $b$-open sets $U$ and $V$ containing $x$ and $y$, respectively such that $y \notin U$ and $x \notin V$;

(ii) $b-T_{2}[2]$ if for each pair of distinct points $x$ and $y$ of $X$, there exist $b$-open sets $U$ and $V$ such that $x \in U, y \in V$ and $U \cap V=\varnothing$;

(iii) b-regular [6] if for any closed set $F \subset X$ and any point $x \in X \backslash F$, there exists disjoint $b$-open sets $U$ and $V$ such that $x \in U$ and $F \subset V$.

Lemma 2.4. [5] For $\mathbf{H} \in p S(X)$, the following are equivalent:

(i) $\mathbf{H}$ is an ultra $p$-stack;

(ii) If $A \cap H=\emptyset$ for all $H \in \mathbf{H}$, then $A \in \mathbf{H}$;

(iii) $B \in H$ implies $X \backslash B \in H$.

Theorem 2.5. [5] Let $f:(X, \tau) \rightarrow(Y, \sigma)$ be a function and $\mathbf{H} \in p S(X)$.

(i) If $\mathbf{H}$ is a filter, so is $f(\mathbf{H})$;

(ii) If $\mathbf{H}$ is a ultra filter, so is $f(\mathbf{H})$;

(iii) If $\mathbf{H}$ is a ultra $p$-stack, so is $f(\mathbf{H})$.

\section{3. $b$-Convergence of $p$-Stacks}

Definition 3.1. Let $X$ be a topological space, $x \in X$ and let $\mathbf{B}(x)=$ $\{V \subset X: V$ is a $b$-neighbourhood of $x\}$. Then we call the family $\mathbf{B}(x)$ the $b$-neighbourhood stack at $x$.

Definition 3.2. Let $X$ be a topological space, $x \in X$ and let $\mathbf{B}(x)=$ $\{V \subset X: V$ is a $b$-neighbourhood of $x\}$. Then we call the family $\mathbf{B}(x)$ the $b$-neighbourhood stack at $x$.

Theorem 3.3. Let $f:(X, \tau)$, be a topological space. Then we have the following

(i) $\dot{x} b$-converges to $x$ for all $x \in X$; 
(ii) If $\mathbf{F} b$-converges to $x$ and $\mathbf{F} \subset \mathbf{G}$ for $\mathbf{F} ; \mathbf{G} \in p S(X)$, then $\mathbf{G} b$-converges to $x$;

(iii) If both $\mathbf{F}$ and $\mathbf{G}$ are $p$-stacks $b$-converging to $x$, then $\mathbf{F} \cap \mathbf{G}$ b-converges to $x$;

(iv) If $p$-stacks $\mathbf{F}_{i} b$-converge to $x$ for all $i \in J$, then $\cap \mathbf{F}_{i}$ b-converges to $x$.

Proof. Follows from the definitions.

Theorem 3.4. Let $(X, \tau)$ be a topological space and $A \subset X$. Then the following are equivalent:

(i) $x \in b \mathrm{Cl}(A)$

(ii) There is $\mathbf{F} \in p S(X)$ such that $A \in \mathbf{F}$ and $\mathbf{F} b$-converges to $\mathrm{x}$;

(iii) For all $V \in \mathbf{B}(x), A \cap V=\emptyset$.

Proof. (i) $\Rightarrow$ (ii): Let $x$ be an element in $b \mathrm{Cl}(A)$, then $U(x) \cap A=\emptyset$ for each $b$-open $U(x)$ of $x$. Let $\mathbf{F}=\mathbf{B}(x) \cup\langle A\rangle$. Then the $p$-stack $\mathbf{F} b$-converges to $x$ and $A \in \mathbf{F}$. (ii) $\Rightarrow$ (iii): Let $F$ be a $p$-stack and $A \in \mathbf{F}$ and $p$-stack $\mathbf{F} b$-converge to $x$. Then $\mathbf{B}(x) \subset F$. Thus since $\mathbf{B}(x)$ is a $p$-stack, we get $U \cap A \neq \emptyset$ for all $U \in B(x)$. (iii) $\Rightarrow(\mathrm{i})$ : It is obvious.

Theorem 3.5. Let $(X, \tau)$ be a topological space and $A \subset X$. Then the following are equivalent:

(i) $x \in b \operatorname{Int}(A)$

(ii) For every $p$-stack $\mathbf{F} b$-converging to $x, A \in \mathbf{F}$;

(iii) $A \in \mathbf{B}(x)$.

Proof. (i) $\Rightarrow$ (ii): Let $x$ be an element in $b \operatorname{Int}(A)$, and let $\mathbf{F} b$-stack $b$ converges to $x$. Since $x \in b \operatorname{Int}(A)$, there is a $b$-open subset $U$ such that $x \in$ $U \subset A$, so $A \in \mathbf{B}(x)$. Thus by the definition of $b$-convergence of $p$-stack, we can say $A \in \mathcal{F}$. (ii) $\Rightarrow$ (iii): The $b$-neighborhood stack $\mathbf{B}(x)$ is always $b$-converges to $x$. Thus by (ii), $A \in \mathbf{B}(x)$. (iii) $\Rightarrow$ (i): It is obvious.

Now by using $b$-convergence of $p$-stacks, we characterize the properties of $b-T_{1}, b-T_{2}$ and $b$-regular induced by $b$-open subsets on a topological space.

Theorem 3.6. Let $(X, \tau)$ be a topological space. Then the following statements are equivalent: 
(i) $(X, \tau)$ is $b-T_{1}$

(ii) $\cap \mathbf{B}(x)=\{x\}$ for $x \in X$

(iii) if $\dot{x} b$-converges to $y$, then $x=y$.

Proof. (i) $\Rightarrow$ (ii): Let $y$ be an element in $\cap \mathbf{B}(x)$, then $y \in U$ for each $b$ open neighborhood $U$ of $x$. Since $X$ is $b-T_{1}$, we get $y=x$. (ii) $\Rightarrow$ (iii): Let $\dot{x} b$-converge to $y$. Since $\mathbf{B}(y) \subset x, x$ is an element in $\cap \mathbf{B}(y)$. Thus $x=y$. (iii) $\Rightarrow(\mathrm{i})$ : Suppose that $X$ is not $b-T_{1}$, then there are distinct $x$ and $y$ such that every $b$-open neighborhood of $x$ contains $y$. Thus $\mathbf{B}(x) \subset \dot{y}$ and $\dot{y} b$-converges to $x$. This contradicts the hypothesis.

Theorem 3.7. Let $(X, \tau)$ be a topological space. Then the following statements are equivalent:

(i) $(X, \tau)$ is $b-T_{2}$;

(ii) Every b-convergent $p$-stack $\mathbf{F}$ on $X$ b-converges to exactly one point;

(iii) Every b-convergent ultra $p$-stack $\mathbf{F}$ on $X$ b-converges to exactly one point.

Proof. (i) $\Rightarrow\left(\right.$ ii): Suppose that $X$ is $b-T_{2}$ and a $p$-stack $\mathbf{F} b$-converges to $x$. For any $y \neq x$, there are disjoint $b$-open sets $U(x)$ and $U(y)$ containing $x$ and $y$, respectively. Since $\mathbf{B}(x) \subset F$ and $\mathbf{F}$ is a $p$-stack, both $U(x)$ and $X \backslash U(y)$ are elements of $\mathbf{F}$. Thus $\mathbf{F}$ is not finer than $(y)$, so $\mathbf{F}$ doesn't $b$-converge to $y$. (ii) $\Rightarrow$ (iii): It is obvious. (iii) $\Rightarrow(i)$ : Suppose that $X$ is not $b-T_{2}$. Then there must exist $x, y$ such that $U(x) \cap U(y) \neq \emptyset$ for every $b$-open sets $U(x)$ and $U(y)$ of $x$ and $y$, respectively. Let $\mathbf{F}$ be a ultra $p$-stack finer than a $p$-stack $\mathbf{B}(x) \subset B(y)$. Then $\mathbf{F}$ is finer than $B(x)$ and $(y)$, so the ultra $p$-stack $F$-converges to both $x$ and $y$. This contradicts $(i i)$.

If $(X, \tau)$ is a topological space and $F \in p S(X)$, then $\mathbf{B}=\{b \mathrm{Cl}(F)\}: F \in \mathbf{F}$ is a $p$-stack base on $X$, and the $b$-closure $p$-stack generated by $B$ is denoted by $b \mathrm{Cl}(\mathbf{F})$.

Theorem 3.8. Let $(X, \tau)$ be a topological space. Then the following statements are equivalent:

(i) $(X, \tau)$ is b-regular;

(ii) For every $x \in X, \mathbf{B}(x)=b \mathrm{Cl}(\mathbf{B}(x))$;

(iii) If a $p$-stack $F$-converges to $x$, then the $b$-closure $p$-stack $b \mathrm{Cl}(\mathbf{F}) b$-converges to $x$. 
Proof. $(\mathrm{i}) \Rightarrow(i i)$ : Let $F$ be an element in $\mathbf{B}(x)$. There exists a $b$-open neighborhood $U(x)$ such that $U(x) \subset F$. Since $X$ is $b$-regular, there is a $b$-open neighborhood $W(x)$ of $x$ such that $W(x) \subset b \mathrm{Cl}(W(x)) \subset U(x) \subset F$. Since $b \mathrm{Cl}(W(x)) \in b \mathrm{Cl}(\mathbf{B}(x))$ and $b \mathrm{Cl}(\mathbf{B}(x))$ is a $p$-stack, $F \in b \mathrm{Cl}(\mathbf{B}(x))$. $($ ii $) \Rightarrow($ iii $)$ : Let a $p$-stack $\mathbf{F} b$-converge to $x$. Then $\mathbf{B}(x) \subset F$, and so $b \mathrm{Cl}(\mathbf{B}(x)) \subset b \mathrm{Cl}(\mathbf{F})$. By $(i i)$, we get that $b \mathrm{Cl}(\mathbf{F}) b$-converges to $x$. $(i i i) \Rightarrow(i)$ : Let $U$ be a $b$-open set containing $x \in X$. Since $\mathbf{B}(x) b$-converges to $x$, by (iii) $b \mathrm{Cl}(\mathbf{B}(x)) b$-converges to $x$, and so $U \in b \mathrm{Cl}(\mathbf{B}(x))$. Then by the definition of the $b$-closure of $p$-stacks, we can get a $b$-open neighborhood $V$ of $x$ such that $V \subset b \mathrm{Cl}(V) \subset U$.

Definition 3.9. A function $f:(X, \tau) \rightarrow(Y, \sigma)$ is said to be $b$-irresolute [4] if $f^{-1}(V)$ is $b$-closed (resp. $b$-open) in $X$ for every $b$-closed (resp. $b$-open) subset $V$ of $Y$

Theorem 3.10. Let $X$ and $Y$ be topological spaces. Then a function $f:(X, \tau) \rightarrow(Y, \sigma)$ is $b$-irresolute if and only if for each $x$ in $X$ and each $b$ neighborhood $U$ of $f(x)$, there is a b-neighborhood $V$ of $x$ such that $f(V) \subset U$.

Now we get another characterization of the b-irresolute function on a topological space using the notion of $p$-stacks.

Theorem 3.11. For a function $f:(X, \tau) \rightarrow(Y, \sigma)$, the following statements are equivalent:

(i) $f$ is b-irresolute;

(ii) $\mathbf{B}(f(x)) \subset f(\mathbf{B}(x))$ for all $x \in X$;

(iii) If a $p$-stack $\mathbf{F} b$-converges to $x$, then the image $p$-stack $f(\mathbf{F}) b$-converges to $f(x)$.

Proof. $(i) \Rightarrow($ ii $)$ : Let $V$ be any member of $\mathbf{B}(f(x))$ in $Y$. Then there is a $b$-open set $W$ such that $W \subset V$. Since $f$ is $b$-irresolute, there exists a $b$-open neighborhood $U \in \mathbf{B}(x)$ such that $f(U) \subset W \subset V$, thus $V \in f(\mathbf{B}(x))$. $(i i) \Rightarrow($ iii $)$ : It is obvious. $($ iii $) \Rightarrow(i)$ : If $f$ is not $b$-irresolute, then for some $x \in X$, there is a $b$-open neighborhood $V \in \mathbf{B}(f(x))$ such that for all $b$-open neighborhood $U \in \mathbf{B}(x), f(U)$ is not included in $V$. For all $U \in \mathbf{B}(x)$, since $f(U) \cap(Y \backslash V) \neq \emptyset$, we get a $p$-stack $\mathbf{F}=f(\mathbf{B}(x)) \cup\langle Y \backslash V>$. And since $U \cap f^{-1}(Y \backslash V) \neq \emptyset$, also we get a $p$-stack $\mathbf{G}=\mathbf{B}(x) \cup f^{-1}<Y \backslash V>$ which $b$-converges to $x$.But since $f(\mathbf{G})$ is a finer $p$-stack than $F$ and $Y \backslash V \in \mathbf{F}, f(\mathbf{G})$ 
can't b-converge to $f(x)$, contradicting to $(i i i)$.

Now we introduce a new notion of $p$-b-compactness by $p$-stacks and investigate the related properties.

Definition 3.12. Let $(X, \tau)$ be a topological space and $A$ be a subset of $X$. A subset $A$ of a topological space $(X, \tau)$ is $p$-b-compact if every ultra $p$-stack containing $A b$-converges to a point in $A$. A topological space $(X, \tau)$ is $p$-b-compact if $X$ is $p$-b-compact.

Let $X=\{a, b, c\}$. In case $\tau$ is the discrete topology, let $\mathbf{H}$ be an ultra $p$-stack containing a $p$-stack $\mathbf{F}$ generated by $\{\{\mathrm{a}, \mathrm{b}\},\{\mathrm{b}, \mathrm{c}\},\{\mathrm{a}, \mathrm{c}\}\}$. Then it does not $b$-converge to any point in $X$. Thus the topological space $(X, \tau)$ is not $p$-b-compact. But in case $\tau=\{\emptyset,\{a\},\{b, c\}, X\}$ the topological space $(X, \tau)$ is $p$-b-compact.

Theorem 3.13. If a topological space $(X, \tau)$ is $p$-b-compact and $A \subset X$ is $b$-closed, then $A$ is $p$-b-compact.

Proof. Let $\mathbf{F}$ be an ultra $p$-stack containing $A$. From Definition 3.12, there is $x \in X$ such that $\mathbf{F} b$-converges to $x$. Thus $\mathbf{B}(x) \subset F$, and since $A \in \mathbf{F}$ and $\mathbf{F}$ is a $p$-stack, $A \cap V \neq \emptyset$ for all $V \in \mathbf{B}(x)$. So by Theorem 9, we can say $x \in b \mathrm{Cl}(A)=A$.

Theorem 3.14. The b-irresolute image of a $p$-b-compact set is $p$-b-compact.

Proof. Let a function $f:(X, \tau) \rightarrow(Y, \sigma)$ be $b$-irresolute, let $A \subset X$ be $p$-b-compact, and let $\mathbf{H}$ be an ultra $p$-stack containing $f(A)$. If $\mathbf{G}$ is an ultra $p$-stack containing the $p$-stack base $\left\{f^{-1}(H): H \in \mathbf{H}\right\} \cup\langle A\rangle$, then for some $x \in A, \mathbf{G} b$-converges to $x$, and $\mathbf{H}=f(\mathbf{G}) b$-converges to $f(x)$. Thus, $f(A)$ is $p$-b-compact.

Theorem 3.15. A topological space $(X, \tau)$ is $p$-b-compact if and only if each $b$-open cover of $X$ has a two-element subcover.

Proof. Suppose $\mathbf{H}$ is an ultra $p$-stack in $X$ such that it does not $b$-converge to any point in $X$. Then for each $x \in X$, there is a $b$-open subset $U_{x} \in \mathbf{B}(x)$ such that $U_{x} \notin H$. By Lemma 2.4(iii), $X \backslash U_{x} \in H$, for all $x \in X$. Thus $\mathbf{U}=\left\{U_{x}: x \in X\right\}$ is a $b$-open cover of $X$. But $\mathbf{U}$ has no two-element subcover 
of $X$, for if $U, V \in \mathbf{U}$ and $X \subset U \cup V$, then $(X \backslash U) \cap(X \backslash V)=X \backslash(U \cup V)=\emptyset$, contradicting the assumption that $\mathbf{H}$ is a $p$-stack. Conversely, let $\mathbf{U}$ be a $b$-open cover of $X$ with no two-element subcover of $X$. Then $\mathbf{B}=\{X \backslash U: U \in \mathbf{U}\}$ is $p$-stack base, and any ultra $p$-stack containing $\mathbf{B}$ cannot $b$-converge to any point in $X$.

\section{References}

[1] D. Andrijevic, On b-open sets, Math. Vesnik, 48 (1996), 59-64.

[2] E. Ekici, On R-spaces, Int. J. Pure. Appl. Math., 25, No. 2 (2005), 163-172.

[3] M. Caldas, S. Jafari and T. Noiri, On $\wedge_{b}$-sets and the associated topology $\tau^{\wedge_{b}}$, Acta. Math. Hungar., 110, No. 4 (2006), 337-345.

[4] A. A. El-Atik, A Study of Some Types of Mappings on Topological Spaces, Master's Thesis, Faculty of Science, Tanta University, Tanta, Egypt (1997).

[5] D.C. Kent, W.K. Min, Neighbourhood spaces, Int. J. Math. Math. Sci., 32, No. 7 (2002), 387-399.

[6] J.H. Park, Strongly $\theta$-b-continuous functions, Acta Math. Hungar., 110, No. 4 (2006), 347-359. 